\title{
Bielliptic modular curves $X_{1}(N)$
}

\author{
by \\ Daeyeol Jeon and Chang Heon Kim (Seoul)
}

0. Introduction. Let $\Gamma=\mathrm{SL}_{2}(\mathbb{Z})$ be the full modular group. For any integer $N \geq 1$, we have subgroups $\Gamma(N), \Gamma_{1}(N), \Gamma_{0}(N)$ of $\Gamma$ defined by matrices $\left(\begin{array}{ll}a & b \\ c & d\end{array}\right)$ congruent modulo $N$ to

$$
\left(\begin{array}{ll}
1 & 0 \\
0 & 1
\end{array}\right), \quad\left(\begin{array}{ll}
1 & * \\
0 & 1
\end{array}\right), \quad\left(\begin{array}{ll}
* & * \\
0 & *
\end{array}\right)
$$

respectively. We let $X(N), X_{1}(N), X_{0}(N)$ be the modular curves defined over $\mathbb{Q}$ associated to $\Gamma(N), \Gamma_{1}(N), \Gamma_{0}(N)$ respectively. The $X$ 's are compact Riemann surfaces. Denote the genera of $X_{1}(N), X_{0}(N)$ by $g_{1}(N), g_{0}(N)$ respectively.

A smooth, projective curve $X$ with genus $g(X) \geq 2$ is called hyperelliptic (respectively bielliptic) if it admits a map $\phi: X \rightarrow C$ of degree 2 onto a curve $C$ of genus zero (respectively one).

Harris and Silverman [H-S] showed that if a curve $X$ with $g(X) \geq 2$ defined over a number field $K$ is neither hyperelliptic nor bielliptic, then the set of quadratic points on $X$,

$$
\{P \in X(\bar{K}):[K(P): K] \leq 2\}
$$

is finite.

Bars [B] determined all the bielliptic modular curves of type $X_{0}(N)$ and also found all curves $X_{0}(N)$ which have infinitely many quadratic points over $\mathbb{Q}$.

In this paper, we shall determine all the bielliptic modular curves of type $X_{1}(N)$. Our result is as follows.

TheOREM 0.1. The curve $X_{1}(N)$ is bielliptic for exactly 8 values of $N$, namely for $N=13,16,17,18,20,21,22,24$.

2000 Mathematics Subject Classification: 11G18, 11G30.

The first author supported by the Brain Korea 21 Project in 2001.

The work of the second author was supported by the Post-doctoral Fellowship Program of Korea Science and Engineering Foundation (KOSEF). 
We also discuss the problem of determining all modular curves $X_{1}(N)$ which have infinitely many quadratic points over $\mathbb{Q}$.

1. Preliminaries. Let $\Delta$ be a subgroup of $(\mathbb{Z} / N \mathbb{Z})^{*}$. Let $X_{\Delta}(N)$ be the modular curve defined over $\mathbb{Q}$ associated to the modular group $\Gamma_{\Delta}(N)$ :

$$
\Gamma_{\Delta}(N)=\left\{\left(\begin{array}{ll}
a & b \\
c & d
\end{array}\right) \in \Gamma \mid c \equiv 0 \bmod N,(a \bmod N) \in \Delta\right\} .
$$

We always assume that $-1 \in \Delta$. For $d \mid N$, let $\pi_{d}$ be the natural projection from $(\mathbb{Z} / N \mathbb{Z})^{*}$ to $(\mathbb{Z} /\{d, N / d\} \mathbb{Z})^{*}$, where $\{d, N / d\}$ is the least common multiple of $d$ and $N / d$.

TheOrem $1.1([\mathrm{~K}])$. The genus of the modular curve $X_{\Delta}(N)$ is

$$
g\left(X_{\Delta}(N)\right)=1+\frac{\mu}{12}-\frac{\nu_{2}}{4}-\frac{\nu_{3}}{3}-\frac{\nu_{\infty}}{2},
$$

where

$$
\begin{aligned}
\mu & =N \prod_{\substack{p \mid N \\
\text { prime }}}\left(1+\frac{1}{p}\right) \frac{\varphi(N)}{|\Delta|}, \\
\nu_{2} & =\left|\left\{(b \bmod N) \in \Delta \mid b^{2}+1 \equiv 0 \bmod N\right\}\right| \cdot \frac{\varphi(N)}{|\Delta|}, \\
\nu_{3} & =\left|\left\{(b \bmod N) \in \Delta \mid b^{2}-b+1 \equiv 0 \bmod N\right\}\right| \cdot \frac{\varphi(N)}{|\Delta|}, \\
\nu_{\infty} & =\sum_{\substack{d \mid N \\
d>0}} \frac{\varphi(d) \cdot \varphi(N / d)}{\left|\pi_{d}(\Delta)\right|} .
\end{aligned}
$$

Proposition 1.2. Let $v$ be any involution on the compact Riemann surface $X$, and let \# denote the number of fixed points of $v$. Then we have the following genus formula:

$$
g(v \backslash X)=\frac{1}{4}(2 g(X)+2-\#) .
$$

Proof. This follows from the Hurwitz formula.

For an integer $a$ prime to $N$, let $[a]$ denote the automorphism of $X_{1}(N)$ represented by $\gamma \in \Gamma_{0}(N)$ such that $\gamma \equiv\left(\begin{array}{l}a \\ 0\end{array} *\right.$ * $)$ mod $N$. Sometimes we regard $[a]$ as a matrix.

For any matrices $A, B \in M_{2}(\mathbb{Z})$ which give automorphisms on $X_{1}(N)$, we write $A \equiv B \bmod \Gamma_{1}(N)$ if $A^{-1} B \in \pm \Gamma_{1}(N)$. In fact, if $A \equiv B \bmod \Gamma_{1}(N)$, then $A$ and $B$ define the same automorphism on $X_{1}(N)$.

For each divisor $d \mid N$ with $(d, N / d)=1$, consider the matrices of the form 


$$
\left(\begin{array}{cc}
d x & y \\
N z & d w
\end{array}\right)
$$

with $x, y, z, w \in \mathbb{Z}$ and determinant $d$. They define a unique involution on $X_{0}(N)$, called the Atkin-Lehner involution and denoted by $W_{d}$. In particular, if $d=N$, then $W_{N}$ is called the full Atkin-Lehner involution. We also denote by $W_{d}$ a matrix of the above form.

Now we fix a matrix $W_{d}$. By $[\mathrm{K}-\mathrm{Ko} 2], W_{d}$ belongs to the normalizer of $\Gamma_{1}(N)$ in $\mathrm{PSL}_{2}(\mathbb{R})$ and therefore defines an automorphism of $X_{1}(N)$. For each integer $a$ prime to $N,[a] W_{d}$ defines a different automorphism of $X_{1}(N)$. Furthermore $W_{d}$, in general, does not give an involution on $X_{1}(N)$. But when $d=N, W_{N}$ still gives an involution on $X_{1}(N)$ whose properties are investigated in the following proposition.

Proposition 1.3. Let $\psi: X_{1}(N) \rightarrow X_{0}(N)$ be the Galois covering with Galois group $G=(\mathbb{Z} / N \mathbb{Z})^{*} / \pm 1$.

(1) $W_{N}$ defines an involution on $X_{1}(N)$ and $[a] W_{N} \equiv W_{N}\left[a^{-1}\right] \bmod$ $\Gamma_{1}(N)$ for each $a \in G$.

(2) Let $\tau_{0} \in X_{0}(N)$ be a fixed point of $W_{N}$. Then the covering $\psi$ is unramified at each inverse image of $\tau_{0}$. Thus the number of inverse images of $\tau_{0}$ is equal to the degree of $\psi$.

(3) Let $\tau \in X_{1}(N)$ be a fixed point of $W_{N}$. For each $a \in G,[a] \tau$ is also fixed by $W_{N}$ if and only if $a^{2} \equiv \pm 1 \bmod N$.

(4) Let $c \in G \backslash G^{2}$ and $\tau, \tau^{\prime} \in X_{1}(N)$ be fixed by $W_{N}$ and $[c] W_{N}$ respectively. Then $\psi(\tau) \neq \psi\left(\tau^{\prime}\right)$.

Proof. (1) We can write $W_{N}=[b]\left(\begin{array}{cc}0 & -1 \\ N & 0\end{array}\right)$ for some $b \in G$. It is easy to check that $[b]\left(\begin{array}{cc}0 & -1 \\ N & 0\end{array}\right) \equiv\left(\begin{array}{cc}0 & -1 \\ N & 0\end{array}\right)\left[b^{-1}\right] \bmod \Gamma_{1}(N)$. Thus $W_{N}^{2} \equiv\left(\begin{array}{cc}0 & -1 \\ N & 0\end{array}\right)^{2} \bmod$ $\Gamma_{1}(N)$ defines the identity map on $X_{1}(N)$ and the relation $[a] W_{N} \equiv W_{N}\left[a^{-1}\right]$ $\bmod \Gamma_{1}(N)$ is satisfied.

(2) If $1 \leq N \leq 4$, then $\psi$ is the trivial covering and thus it is unramified. If $N \geq 5$, then one can show that the coset $\Gamma_{0}(N) W_{N}=\Gamma_{0}(N)\left(\begin{array}{cc}0 & -1 \\ N & 0\end{array}\right)$ has no parabolic elements and can have elliptic elements of order 2. Therefore the fixed points of $W_{N}$ on $X_{0}(N)$ are neither elliptic points nor cusp points. Thus ramification does not occur over those points.

(3) is straightforward.

(4) If $\psi(\tau)=\psi\left(\tau^{\prime}\right)$, then $\tau^{\prime}=[b] \tau$ for some $b \in G$. Thus $[b] W_{N}[b]^{-1} \tau^{\prime}$ $=\tau^{\prime}$. Now $[b]^{2} W_{N}$ turns out to be $[c] W_{N}$. This is a contradiction to $c \in$ $G \backslash G^{2}$.

Corollary 1.4. With the notation of Proposition 1.3 and $N \geq 5$, let $n$ denote the degree of $\psi(=|G|)$. Assume that (1) $n$ is odd or (2) $n$ is even 
and $g_{0}(N) \leq 1$. Then the numbers of fixed points of $W_{N}$ on $X_{0}(N)$ and on $X_{1}(N)$ are the same.

Proof. (1) Let $\tau_{0} \in X_{0}(N)$ be a fixed point of $W_{N}$. By Proposition 1.3(2), there are $n$ distinct points of $X_{1}(N)$ lying over $\tau_{0}$. Since $W_{N}$ permutes these points and $n$ is odd, at least one of them must be fixed. But by Proposition $1.3(3)$, exactly one of them is fixed.

(2) First we consider the case $g_{0}(N)=1$. The matrix $\left(\begin{array}{cc}0 & -1 \\ N & 0\end{array}\right)$ always has a fixed point on the complex upper half plane $\mathcal{H}$, and hence $W_{N}$ always has a fixed point on $X_{0}(N)$. Thus by Proposition $1.2, W_{N}$ fixes exactly four points of $X_{0}(N)$. The fixed points of $W_{N}$ on $X_{1}(N)$ certainly lie over those four points. By a suitable choice of $\gamma \in \Gamma_{1}(N)$ we can form an elliptic element $\gamma W_{N}$. Thus $W_{N}$ has at least one fixed point on $X_{1}(N)$. Except $N=24$, the order of $G / G^{2}$ is 2 . Thus the number of fixed points is less than or equal to 8 . Possible numbers are 4,8,2 or 6. From Proposition 1.2 the latter two are impossible. By Proposition 1.3(4), 8 can also be excluded. If $N=24$, the order of $G / G^{2}$ is 4 . Thus $W_{N}$ has at least four fixed points. But for each $c=5,7,11,[c] W_{N}$ also has at least four fixed points. By Proposition 1.3(4), the image sets of their fixed points cannot intersect and so we are done. The case $g_{0}(N)=0$ can be proved similarly.

By [O1] we have the following description of cusps. The cusps of $X(N)$ can be regarded as pairs $\pm\left(\begin{array}{l}x \\ y\end{array}\right)$, where $x, y \in \mathbb{Z} / N \mathbb{Z}$, and are relatively prime, and $\left(\begin{array}{l}x \\ y\end{array}\right),\left(\begin{array}{c}-x \\ -y\end{array}\right)$ are identified; $\Gamma / \Gamma(N)$ operates naturally on the left, and so a cusp of $X_{0}(N)$ or $X_{1}(N)$ can be regarded as an orbit of $\Gamma_{0}(N) / \Gamma(N)$ or $\Gamma_{1}(N) / \Gamma(N)$. For each $d \mid N$, a cusp of $X_{1}(N)$ is represented by a pair $\left(\begin{array}{l}x \\ y\end{array}\right)$ with $x$ reduced modulo $d=(y, N)$ and $(x, d)=1$. If $g_{1}(N)>0$, then we have $\frac{1}{2} \varphi(d) \varphi(N / d) \operatorname{cusps}\left(\begin{array}{l}x \\ y\end{array}\right)$ with $d=(y, N)$ and the cusps $\left(\begin{array}{l}x \\ y\end{array}\right)$ with a fixed value of $\pm y$ are conjugate, and in particular are rational only if $\varphi(d)=1$, i.e. $d=1$ or 2 . For each $d \mid N$, a cusp of $X_{0}(N)$ is represented by a pair $\left(\begin{array}{l}x \\ d\end{array}\right)$ with $x$ reduced modulo $t=(d, N / d)$. We have $\varphi(t)$ conjugate cusps $\left(\begin{array}{l}x \\ d\end{array}\right)$ corresponding to $d$, each with ramification degree $e=t$ in the Galois covering $X_{1}(N) \rightarrow X_{0}(N)$.

Let $\Gamma_{1}^{*}(N)$ be the normalizer of $\bar{\Gamma}_{1}(N)= \pm \Gamma_{1}(N) / \pm 1$ in $\mathrm{PSL}_{2}(\mathbb{R})$. Let Aut $X_{1}(N)$ be the group of automorphisms of $X_{1}(N)$. In [K-Ko2], Kim and Koo showed that $\Gamma_{1}^{*}(N)$ is generated by $\bar{\Gamma}_{0}(N)=\Gamma_{0}(N) / \pm 1$ and the matrices $W_{d}$ with $d \mid N$ and $(d, N / d)=1$. Also Ishii and Momose [I-M] established that Aut $X_{1}(N)$ is equal to $\Gamma_{1}^{*}(N) / \bar{\Gamma}_{1}(N)$ for hyperelliptic curves $X_{1}(N)$, i.e. $N=13,16,18$. Later for square free $N$, Momose $[\mathrm{M}]$ verified that Aut $X_{1}(N)=\Gamma_{1}^{*}(N) / \bar{\Gamma}_{1}(N)$. Therefore, for such $N$, Aut $X_{1}(N)$ is generated by $\bar{\Gamma}_{0}(N) / \bar{\Gamma}_{1}(N)$ and the automorphisms induced by the matrices $W_{d}$. 
2. Non-bielliptic curves. For the reader's convenience, in Table 1 we tabulate the genera of $X_{1}(N)$ for $1 \leq N \leq 60$ ([K-Ko1]). There is a misprint in the table of [K-Ko1, p. 297]: $g_{1}(18)=3$ should be corrected to $g_{1}(18)=2$.

We assume that $g_{1}(N) \geq 2$, i.e. $N=13$ or $N \geq 16$. We recall that if $X_{1}(N)$ is a bielliptic curve, there exists an involution $v$, called a bielliptic involution, such that $v \backslash X_{1}(N)$ is an elliptic curve. If $g_{1}(N) \geq 6$, by Proposition 1.2 of $[\mathrm{Sch}], v$ is unique, defined over $\mathbb{Q}$, and lies in the center of Aut $X_{1}(N)$. Then either $v$ is contained in the Galois group of $X_{1}(N)$ over $X_{0}(N)$ or it induces an involution $\widetilde{v}$ on $X_{0}(N)$ such that $\widetilde{v} \backslash X_{0}(N)$ is a rational or elliptic curve. In the first case, we must of course have $g_{0}(N) \leq 1$. Now we divide $N$ into 3 cases.

Table 1

\begin{tabular}{cc|cc|cc|cc|cc|cc}
\hline$N$ & $g_{1}(N)$ & $N$ & $g_{1}(N)$ & $N$ & $g_{1}(N)$ & $N$ & $g_{1}(N)$ & $N$ & $g_{1}(N)$ & $N$ & $g_{1}(N)$ \\
\hline 1 & 0 & 11 & 1 & 21 & 5 & 31 & 26 & 41 & 51 & 51 & 65 \\
2 & 0 & 12 & 0 & 22 & 6 & 32 & 17 & 42 & 25 & 52 & 55 \\
3 & 0 & 13 & 2 & 23 & 12 & 33 & 21 & 43 & 57 & 53 & 92 \\
4 & 0 & 14 & 1 & 24 & 5 & 34 & 21 & 44 & 36 & 54 & 52 \\
5 & 0 & 15 & 1 & 25 & 12 & 35 & 25 & 45 & 41 & 55 & 81 \\
6 & 0 & 16 & 2 & 26 & 10 & 36 & 17 & 46 & 45 & 56 & 61 \\
7 & 0 & 17 & 5 & 27 & 13 & 37 & 40 & 47 & 70 & 57 & 85 \\
8 & 0 & 18 & 2 & 28 & 10 & 38 & 28 & 48 & 37 & 58 & 78 \\
9 & 0 & 19 & 7 & 29 & 22 & 39 & 33 & 49 & 69 & 59 & 117 \\
10 & 0 & 20 & 3 & 30 & 9 & 40 & 25 & 50 & 48 & 60 & 57 \\
\hline
\end{tabular}

CASE I: $g_{1}(N)>6$ and $g_{0}(N)=0$ or 1 , i.e. $N=19,25,27,32,36,49$. CASE II: $g_{1}(N)>6$ and $g_{0}(N) \geq 2$.

CASE III: $2 \leq g_{1}(N) \leq 6$, i.e. $N=13,16,17,18,20,21,22,24$.

First we consider the six values of $N$ which belong to Case I.

Lemma 2.1. $X_{1}(19)$ is not a bielliptic curve.

Proof. Note that Aut $X_{1}(19)$ is generated by $\bar{\Gamma}_{0}(19) / \bar{\Gamma}_{1}(19)$ and $W_{19}$. First, there is no involution of type $[a]$. By Proposition 1.2 and Corollary 1.4, we have $g\left(W_{19} \backslash X_{1}(19)\right)=3$. Therefore $W_{19}$ is not a bielliptic involution.

Lemma 2.2. $X_{1}(27)$ is not a bielliptic curve.

Proof. According to [Ke-M1], the only points on $X_{1}(27)$ that are rational or quadratic over $\mathbb{Q}$ are certain cusps. The bielliptic involution $v$ would be defined over $\mathbb{Q}$ and hence would preserve these points. Let $S_{d}$ be the set of $\Gamma_{1}(N)$-inequivalent cusps $\left(\begin{array}{l}x \\ y\end{array}\right)$ with $(y, N)=d$. Then $S_{1}$ (resp. $\left.S_{3}\right)$ consists of rational (resp. quadratic) cusps and it is not changed by $v$. Under an 
involution $W_{27}$, the set $S_{1}$ (resp. $S_{3}$ ) is mapped to $S_{27}$ (resp. $S_{9}$ ). Since $v$ commutes with $W_{27}$, all cusps in $S_{9}$ or $S_{27}$ are also preserved by $v$. Thus $v$ induces an automorphism of $Y_{1}(27)$ and so comes from an element in the normalizer of $\Gamma_{1}(27)$. First, there is no involution of $X_{1}(27)$ of type $[a]$. By Proposition 1.2 and Corollary 1.4, $g\left(W_{27} \backslash X_{1}(27)\right)=6$. Thus $W_{27}$ is not a bielliptic involution.

Lemma 2.3. $X_{1}(25)$ and $X_{1}(32)$ are not bielliptic.

Proof. Note that [7] (resp. [15]) induces an involution on $X_{1}(25)$ (resp. $X_{1}(32)$ ). By Theorem 1.1, the genus of $[7] \backslash X_{1}(25)$ (resp. [15] $\backslash X_{1}(32)$ ) is 4 (resp. 5) and $g_{1}(25)=12$ (resp. $g_{1}(32)=17$ ). By Proposition 1.2, [7] (resp. [15]) has 10 (resp. 16) fixed points. However, if a curve of genus at least 6 has an involution with more than 8 fixed points, then by Proposition 1.2(b) of [Sch] either this involution is the bielliptic involution or the curve is not bielliptic. Now the assertion follows immediately.

Lemma 2.4. $X_{1}(36)$ and $X_{1}(49)$ are not bielliptic.

Proof. Suppose that $X_{1}(36)$ is bielliptic with bielliptic involution $v$. From Theorem 1.1 one can check that $v$ does not belong to the Galois group of $X_{1}(36)$ over $X_{0}(36)$. Let $\widetilde{v}$ be the involution on $X_{0}(36)$ induced by $v$. Note that $g_{0}(36)=1$ and $g\left(\widetilde{v} \backslash X_{0}(36)\right)=0$. By Proposition 1.2, $\widetilde{v}$ has 4 fixed points. Since the degree of the covering $X_{1}(36) \rightarrow X_{0}(36)$ is equal to 6 , there are 24 fixed points of $v$ in $X_{1}(36)$. But this contradicts Proposition 1.2. Thus $X_{1}(36)$ is not bielliptic. Similarly, $X_{1}(49)$ is not bielliptic, either.

Now we consider Case II. The image of a bielliptic curve under a finite morphism of curves is either bielliptic, hyperelliptic, elliptic or rational (see $[\mathrm{H}-\mathrm{S}])$. Since there is a finite morphism $X_{1}(N) \rightarrow X_{0}(N)$, we have

Lemma 2.5 (Corollary 3.16 of [B]). The modular curves $X_{1}(N)$ are not bielliptic for $N \geq 132$ and for all $N$ in the table below:

$52,57,58,66,67,68,70,73,74,76,77,78,80,82,84,85$, $86,87,88,90,91,93,96,97,98,99,100,102,103,104,105$, $106,107,108,109,110,111,112,113,114,115,116,117$, $118,120,121,122,123,124,125,126,127,128,129,130$.

Lemma 2.6. $X_{1}(N)$ is not a bielliptic curve for the following $N$ :

$23,29,31,41,43,47,53,59,61,65,71,75,79,83,89,95$, $101,119,131$.

Proof. Let $N$ be one of the numbers of the above list. Then the curve $X_{0}(N)$ is either hyperelliptic or bielliptic, but not both. From the tables in [B, O2], we know that the hyperelliptic or bielliptic involution is the full Atkin-Lehner involution. Suppose that $X_{1}(N)$ is bielliptic and let $v$ be the 
bielliptic involution. Since $g_{0}(N) \geq 2$, the involution $v$ induces an involution $\widetilde{v}$ on $X_{0}(N)$ which is the full Atkin-Lehner involution. Then $\widetilde{v}$ maps the cusp $\left(\begin{array}{l}0 \\ 1\end{array}\right)$ to $\left(\begin{array}{l}1 \\ 0\end{array}\right)$. Thus $v$ maps the cusps lying above $\left(\begin{array}{l}0 \\ 1\end{array}\right)$ to the cusps lying above $\left(\begin{array}{l}1 \\ 0\end{array}\right)$. Note that the cusps over $\left(\begin{array}{l}0 \\ 1\end{array}\right)$ are rational but the cusps over $\left(\begin{array}{l}1 \\ 0\end{array}\right)$ are non-rational. This is a contradiction.

Lemma 2.7. $X_{1}(N)$ is not a bielliptic curve for the following $N$ :

$30,33,35,38,39,42,46,51,55,60,62,69,92,94$.

Proof. Let $N$ be one of the numbers of the above list. From the tables in [B, O2], we know that any hyperelliptic or bielliptic involution on $X_{0}(N)$ is equal to one of the Atkin-Lehner involutions $W_{d}$ with $d \neq 2$. Suppose that $X_{1}(N)$ is bielliptic and $v$ is the bielliptic involution. Then $v$ induces an involution $\widetilde{v}$ on $X_{0}(N)$ which is $W_{d}$ with $d \neq 2$. Note that $W_{d}$ is represented by a matrix $\left(\begin{array}{cc}d x & y \\ N z & d w\end{array}\right)$ where $x, y, z, w \in \mathbb{Z}$ and $\operatorname{det} W_{d}=d$. We can choose $w=1$ and $(y, d)=1$. Then $\widetilde{v}$ maps the cusp $\left(\begin{array}{l}0 \\ 1\end{array}\right)$ to $\left(\begin{array}{l}y \\ d\end{array}\right)$. Since the cusps lying above $\left(\begin{array}{l}y \\ d\end{array}\right)$ are non-rational, $v$ maps rational cusps to non-rational cusps. This gives rise to a contradiction.

Lemma 2.8. $X_{1}(N)$ is not a bielliptic curve for the following $N$ :

$26,28,34,40,44,45,48,50,54,56,64,72,81$.

Proof. Let $N$ be one of the numbers of the above list. Suppose that $X_{1}(N)$ is a bielliptic curve with bielliptic involution $v$. Let $\widetilde{v}$ be the induced involution on $X_{0}(N)$. Since $N \neq 37,63$, every automorphism of $X_{0}(N)$ is a modular automorphism (see [Ke-M2]). So for our 13 values of $N$, the possible candidate for $\widetilde{v}$ is also a modular automorphism. Thus $v$ is induced from an element of $\Gamma_{1}^{*}(N)$. If $v$ is $W_{d}$ with $d \neq 2$, we are done by the same arguments as in Lemmas 2.6 and 2.7. For example, if $N=40, v$ can be one of $W_{5}, W_{8}, W_{40}$ and so we can apply the rationality argument.

If $N=54, v$ cannot be $W_{2}$ since the genus of $W_{2} \backslash X_{0}(56)$ is 2 . For $N=26,34,50, v$ may happen to be $W_{2}$. In these 3 cases we can use the counting argument used in the proof of Lemma 2.4 to show that $X_{1}(N)$ is not bielliptic, either.

Lemma 2.9. $X_{1}(37)$ and $X_{1}(63)$ are not bielliptic curves.

Proof. Let $N$ be 37 or 63 . Suppose that $X_{1}(N)$ is bielliptic and $v$ is the bielliptic involution. Let $\widetilde{v}$ be the induced involution on $X_{0}(N)$.

If $N=37$, Aut $X_{1}(37)$ is generated by $\bar{\Gamma}_{0}(37) / \bar{\Gamma}_{1}(37)$ and $W_{37}$ because 37 is square free. Thus the involution $\widetilde{v}$ must be a modular automorphism, and hence equal to $W_{37}$. By the same argument as in the proof of Lemma 2.6 , this is a contradiction. 
If $N=63$, the involution $\widetilde{v}$ must be a bielliptic involution. We can deal with this case by applying the counting argument used in the proof Lemma 2.4 to show that $X_{1}(63)$ is not bielliptic, either.

3. Bielliptic curves. In this section we will show that for all values of $N$ in Case III, $X_{1}(N)$ is bielliptic.

Lemma 3.1. $X_{1}(N)$ is a bielliptic curve for $N=13,16,18,20$.

Table 2

\begin{tabular}{|c|c|}
\hline$N$ & Some bielliptic involutions \\
\hline 13 & {$[a]\left(\begin{array}{cc}0 & -1 \\
13 & 0\end{array}\right) \quad(a=1, \ldots, 6)$} \\
\hline 16 & $(a=1,3,5,7)$ \\
\hline 18 & {$[a]\left(\begin{array}{cc}0 & -1 \\
18 & 0\end{array}\right), \quad[7 a] W_{2}\left(\begin{array}{cc}0 & -1 \\
18 & 0\end{array}\right) \quad(a=1,5,7)$} \\
\hline 20 & {$[9], \quad[a]\left(\begin{array}{cc}0 & -1 \\
20 & 0\end{array}\right)$} \\
\hline
\end{tabular}

Proof. From Proposition 1.2 and Corollary 1.4, it follows that $W_{N}=$ $[a]\left(\begin{array}{cc}0 & -1 \\ N & 0\end{array}\right)$ is a bielliptic involution of $X_{1}(N)$ for $N=13,16,18,20$.

For $N=13,16,18$, the curves $X_{1}(13), X_{1}(16), X_{1}(18)$ have genus 2 and so they are hyperelliptic. The hyperelliptic involution $u$ is unique and given by [5], [7], $W_{2}[7]$, respectively ([I-M]). Because these curves have genus 2 , any other involution $v$ must be bielliptic. But since $u$ commutes with every automorphism, $u v$ will be another bielliptic involution. For $N=20$, since $g\left([9] \backslash X_{1}(20)\right)=g\left(X_{\Delta}(20)\right)=1$ where $\Delta=\{ \pm 1, \pm 9\}$, [9] is also a bielliptic involution of $X_{1}(20)$.

Lemma 3.2. Suppose $N$ is even and congruent to 2 modulo 4. Then $W_{2}[a] \equiv[a] W_{2} \bmod \Gamma_{1}(N)$ for all $a \in(\mathbb{Z} / N \mathbb{Z})^{*}$.

Proof. Say

$$
[a]=\left(\begin{array}{ll}
a & b \\
c & d
\end{array}\right), \quad W_{2}=\left(\begin{array}{cc}
2 x & y \\
N z & 2 w
\end{array}\right) .
$$

By a simple calculation, the $(1,1)$-entry of $W_{2}^{-1}[a]^{-1} W_{2}[a]$ is equal to $2 x w-$ $\frac{1}{2} a^{2} y z N \bmod N$. Since $4 x w-y z N=2,2 x w-\frac{1}{2} a^{2} y z N=1+\frac{1}{2}\left(1-a^{2}\right) y z N \equiv$ $1 \bmod N$.

Consider the case $N=22$. Take $W_{2}=\left(\begin{array}{cc}8 & -3 \\ 22 & -8\end{array}\right)$. Then $W_{2}$ is an elliptic element and gives an involution on $X_{1}(22)$. Thus $W_{2} \tau=\tau$ for some $\tau \in \mathcal{H}$. Note that $W_{2}$ defines a bielliptic involution of $X_{0}(22)$. From Proposition 1.2 , we know that the number of fixed points of $W_{2}$ in $X_{0}(22)$ is 2 . Let $\tau_{1}, \tau_{2}$ be the fixed points with $\tau_{1}=\tau$. Since the degree of the covering $X_{1}(22) \rightarrow X_{0}(22)$ is 5 and this covering is unramified, there are five 
points of $X_{1}(22)$ lying above $\tau_{i}(i=1,2)$. For each $i=1,2$, the five points lying above $\tau_{i}$ are represented by $[d] \tau_{i}$ with $d \in(\mathbb{Z} / 22 \mathbb{Z})^{*}$. By the above lemma,

$$
W_{2}[d] \tau_{1}=[d] W_{2} \tau_{1}=[d] \tau_{1} \quad \text { on } X_{1}(22) .
$$

Thus $W_{2}$ fixes the five points lying above $\tau_{1} . W_{2}$ permutes the five points lying above $\tau_{2}$ so that at least one of them must be fixed. Let $W_{2}$ fix $[d] \tau_{2}$ for some $d \in(\mathbb{Z} / 22 \mathbb{Z})^{*}$. For each $d^{\prime} \in(\mathbb{Z} / 22 \mathbb{Z})^{*}$,

$$
\begin{aligned}
W_{2}\left[d^{\prime}\right] \tau_{2} & =W_{2}\left[d^{\prime}\right][d]^{-1}[d] \tau_{2}=\left[d^{\prime}\right][d]^{-1} W_{2}[d] \tau_{2} \\
& =\left[d^{\prime}\right][d]^{-1}[d] \tau_{2}=\left[d^{\prime}\right] \tau_{2} \quad \text { on } X_{1}(22) .
\end{aligned}
$$

Thus $W_{2}$ fixes exactly 10 points of $X_{1}(22)$. By Proposition $1.2, W_{2}$ must be a bielliptic involution. Moreover since $g_{1}(22)=6, W_{2}$ is a unique bielliptic involution.

Lemma 3.3. $X_{1}(22)$ is a bielliptic curve. $W_{2}=\left(\begin{array}{cc}8 & -3 \\ 22 & -8\end{array}\right)$ is the only bielliptic involution.

Lemma 3.4. $X_{1}(17)$ is a bielliptic curve. [4] is the only bielliptic involution.

Proof. Only [4] is an involution of $X_{1}(17)$ of type [a]. By Theorem 1.1, $g\left([4] \backslash X_{1}(17)\right)=g\left(X_{\Delta}(17)\right)=1$ where $\Delta=\{ \pm 1, \pm 4\}$. Thus [4] is a bielliptic involution of $X_{1}(17)$. By [M] and [K-Ko2], other involutions must be of type $W_{17}$. By Proposition 1.2 and Corollary 1.4, we obtain $g\left(W_{17} \backslash X_{1}(17)\right)=2$. Thus $W_{17}$ is not a bielliptic involution.

Lemma 3.5. $X_{1}(21)$ is a bielliptic curve. All the bielliptic involutions are $W_{3}=\left(\begin{array}{cc}9 & -4 \\ 21 & -9\end{array}\right)$ and $[8] W_{3}$.

Proof. Take $W_{3}=\left(\begin{array}{cc}9 & -4 \\ 21 & -9\end{array}\right)$. Then $W_{3}$ is an elliptic element and it defines an involution on $X_{1}(21)$. For $a=1,2,4,5,8,10$, we have $[a] W_{3} \equiv W_{3}[a] \bmod$ $\Gamma_{1}(21)$. By an argument similar to the proof of Lemma $3.2, W_{3}$ has at least six fixed points on $X_{1}(21)$. By Proposition 1.2, the number of fixed points of $W_{3}$ must be 8 or 12 . Since $X_{1}(21)$ is not a hyperelliptic curve, $W_{3}$ cannot have twelve fixed points. Thus the number of fixed points of $W_{3}$ is 8 and then $W_{3}$ is a bielliptic involution. It can be easily seen that [8] $W_{3}$ also gives an involution on $X_{1}(21)$ and it is the only involution of type $[a] W_{3}$ with $a \neq 1$. We can choose a matrix [8] so that $[8] W_{3}$ is an elliptic element. Similarly $[8] W_{3}$ gives another bielliptic involution.

By [M] and [K-Ko2], other involutions can be of type [a], $W_{7}$ or $W_{21}$. Write $W_{7}=\left(\begin{array}{cc}7 x & y \\ 21 z & 7 w\end{array}\right)$ and assume $\frac{1}{7} W_{7}^{2} \equiv \pm 1 \bmod \Gamma_{1}(21)$. Combined with the condition $\operatorname{det} W_{7}=7$, this leads to a contradiction. So $W_{7}$ cannot give an involution on $X_{1}(21)$.

By Proposition 1.2 and Corollary 1.4, the genus of $W_{21} \backslash X_{1}(21)$ is 2 so that the involution $W_{21}$ cannot be a bielliptic involution. 
Among the types $[a]$, only [8] is an involution of $X_{1}(21)$. By Theorem $1.1, g\left([8] \backslash X_{1}(21)\right)=3$. Thus [8] is not a bielliptic involution.

Lemma 3.6. $X_{1}(24)$ is a bielliptic curve. Among the modular automorphisms, [11] is the only bielliptic involution.

Proof. [5], [7], [11] are all the involutions of type [a]. Put $\Delta_{1}=\{ \pm 1, \pm 5\}$, $\Delta_{2}=\{ \pm 1, \pm 7\}, \Delta_{3}=\{ \pm 1, \pm 11\}$. Then $g\left(X_{\Delta_{1}}(24)\right)=g\left(X_{\Delta_{2}}(24)\right)=3$ and $g\left(X_{\Delta_{3}}(24)\right)=1$. Thus [11] is the only bielliptic involution among the above involutions.

Consider the involutions of types $W_{3}, W_{8}, W_{24}$. By Proposition 1.2 and Corollary 1.4, $W_{24}$ cannot be a bielliptic involution. And $W_{3}$ does not give an involution on $X_{1}(24)$. Write $W_{8}=\left(\begin{array}{cc}8 & -3 \\ 24 & -8\end{array}\right)$. Then $W_{8}$ is an elliptic element and gives an involution. For any $a$ prime to $24, a^{2}$ is congruent to $1 \bmod 24$ so that $[a] W_{8} \equiv W_{8}[a] \bmod \Gamma_{1}(N)$. Thus, for such $a,[a] W_{8}$ defines an involution on $X_{1}(24)$. As in the proof of Lemma 3.2, there are at least four fixed points of $W_{8}$ in $X_{1}(24)$. We can choose a matrix $[a]$ so that $[a] W_{8}$ is an elliptic element for any $a$ prime to 24 . Thus each $[a] W_{8}$ also has at least four fixed points in $X_{1}(24)$. One can show that Proposition 1.3(4) is also valid for $W_{8}$. Thus we conclude that $W_{8}$ has exactly four fixed points and so it cannot be a bielliptic involution.

Summarizing the results of the last two sections, we obtain Theorem 0.1 .

REMARK 3.7. $X_{1}(N)$ is a bielliptic curve if and only if $2 \leq g_{1}(N) \leq 6$.

4. Quadratic points. Let $K$ be a quadratic field over $\mathbb{Q}$ and $E$ an elliptic curve defined over $K$. Denote by $E_{\text {tors }}(K)$ the group of $K$-rational torsion points of $E$. Then one has a complete description of $E_{\mathrm{tors}}(K)$.

Theorem 4.1 ([Ka-Ma],[Ke-M1]). $E_{\mathrm{tors}}(K)$ is isomorphic to one of the following:

(i) $\mathbb{Z} / m \mathbb{Z}$ with $m \leq 16$, or $m=18$,

(ii) $\mathbb{Z} / 2 \mathbb{Z} \times \mathbb{Z} / 2 k \mathbb{Z}$ with $k \leq 6$,

(iii) $\mathbb{Z} / 3 \mathbb{Z} \times \mathbb{Z} / 3$ lZ with $l \leq 2$,

(iv) $\mathbb{Z} / 4 \mathbb{Z} \times \mathbb{Z} / 4 \mathbb{Z}$.

As a corollary we can state the following known result:

THEOREM 4.2. The following are equivalent:

(a) $N \leq 18, N \neq 17$.

(b) $g_{1}(N) \leq 2$.

(c) $X_{1}(N)$ is rational, elliptic or hyperelliptic.

(d) $X_{1}(N)$ has infinitely many quadratic points over $\mathbb{Q}$. 
(e) $X_{1}(N)$ has quadratic points over $\mathbb{Q}$ that are not cusps.

(f) There exist infinitely many non-isomorphic elliptic curves $E$ with a primitive $N$-torsion point $P$ such that $E$ is defined over some quadratic number field $K$ (depending on $E$ and $P$ ) and $P$ is $K$-rational.

(g) There exists at least one elliptic curve $E$ defined over some quadratic number field $K$ with a $K$-rational, primitive $N$-torsion point.

Proof. (a) $\Rightarrow(\mathrm{b}) \Rightarrow(\mathrm{c}) \Rightarrow(\mathrm{d}) \Rightarrow(\mathrm{e}) \Rightarrow(\mathrm{g})$ and $(\mathrm{d}) \Rightarrow(\mathrm{f}) \Rightarrow(\mathrm{g})$ are clear, while $(\mathrm{g}) \Rightarrow$ (a) follows from Theorem 4.1 .

REMARK 4.3. (1) Without the above theorem, our classification of bielliptic curves $X_{1}(N)$ shows that there are only finitely many $N$ (essentially $N<25)$ for which $X_{1}(N)$ can have infinitely many quadratic points over $\mathbb{Q}$.

(2) $13,16,18$ are the only values of $N$ such that $X_{1}(N)$ is a bielliptic curve admitting infinitely many quadratic points over $\mathbb{Q}$.

(3) Since a curve $X$ with $g(X) \geq 2$ has infinitely many quadratic points over $\mathbb{Q}$ if and only if $X$ is a hyperelliptic curve or a bielliptic curve over $\mathbb{Q}$ mapping to an elliptic curve $E$ with positive rank, we deduce that all elliptic curves over $\mathbb{Q}$ doubly covered by $X_{1}(N)(N=17,20,21,22,24)$ have rank zero.

Acknowledgments. We thank Dr. Andreas Schweizer for his kind and valuable suggestions concerning the proofs of the results of this paper.

\section{References}

[B] F. Bars, Bielliptic modular curves, J. Number Theory 76 (1999), 154-165.

[H-S] J. Harris and J. H. Silvermann, Bielliptic curves and symmetric products, Proc. Amer. Math. Soc. 112 (1991), 347-356.

[I-M] N. Ishii and F. Momose, Hyperelliptic modular curves, Tsukuba J. Math. 15 (1991), 413-423.

[Ka-Ma] S. Kamienny and B. Mazur, Rational torsion of prime order in elliptic curves over number fields (with an appendix by A. Granville), Columbia University Number Theory Seminar (New York, 1992), Astérisque 228 (1995), 3, 81-100.

[Ke-M1] M. A. Kenku and F. Momose, Torsion points on elliptic curves defined over quadratic fields, Nagoya Math. J. 109 (1988), 125-149.

[Ke-M2] -, - , Automorphism groups of the modular curves $X_{0}(N)$, Comp. Math. 65 (1988), 51-80.

$[\mathrm{K}] \quad$ C. H. Kim, On the genus of $X_{\Delta}(N)$, preprint.

[K-Ko1] C. H. Kim and J. K. Koo, On the genus of some modular curves of level $N$, Bull. Austral. Math. Soc. 54 (1996), 291-297.

[K-Ko2] - - , The normalizer of $\Gamma_{1}(N)$ in $P S L_{2}(\mathbb{R})$, Comm. Algebra 28 (2000), 5303-5310.

[M] F. Momose, Automorphism groups of the modular curves $X_{\Delta}(N)$, preprint.

[O1] A. Ogg, Rational points on certain elliptic modular curves, in: Proc. Sympos. Pure Math. 24, Amer. Math. Soc., 1973, 221-231.

[O2] - Hyperelliptic modular curves, Bull. Soc. Math. France 102 (1974), 449-462. 
[Sch] A. Schweizer, Bielliptic Drinfeld modular curves, Asian J. Math. 5 (2001), $705-720$.

KIAS 207-43

Cheongnyangni 2-dong

Dongdaemun-gu

Seoul, 130-722 Korea

E-mail: dyjeon@kias.re.kr chkim@kias.re.kr

Received on 21.5.2002

and in revised form on 14.2.2003 